\title{
A strong case for fast stellar rotation at very low metallicities
}

\author{
C. Chiappini ${ }^{1,2}$, R. Hirschi ${ }^{3}$, G. Meynet ${ }^{2}$, S. Ekström ${ }^{2}$, A. Maeder ${ }^{2}$, and F. Matteucci ${ }^{4}$
}

1 Osservatorio Astronomico di Trieste, via G. B. Tiepolo 11, 34131 Trieste, Italia
e-mail: Christina.Chiappini@obs. unige.ch
2 Observatoire Astronomique de l’Université de Genève, 1290 Sauverny, Switzerland
3 Dept. of Physics and Astronomy, University of Basel, 4056 Basel, Switzerland
${ }^{4}$ Dipartimento di Astronomia, Universitá degli Studi di Trieste, via G. B. Tiepolo 11, 34131 Trieste, Italia

Received 16 January 2006 / Accepted 20 February 2006

\section{ABSTRACT}

We investigate the effect of new stellar models taking rotation into account and computing for a metallicity $Z=10^{-8}$ on the chemical evolution of the earliest phases of the Milky Way. These models were computed under the assumption that the ratio of the initial rotation velocity to the critical velocity of stars is roughly constant with metallicity. This naturally leads to faster rotation at lower metallicity, as metal-poor stars are more compact than metal-rich ones. We find that the new $Z=10^{-8}$ stellar yields have a tremendous impact on the nitrogen enrichment of the interstellar medium for $\log (\mathrm{O} / \mathrm{H})+12<7$ (or $[\mathrm{Fe} / \mathrm{H}]<-3$ ). We show that including $Z=10^{-8}$ stellar yields in chemical evolution models, both high $\mathrm{N} / \mathrm{O}$ and $\mathrm{C} / \mathrm{O}$ ratios are obtained in the very-metal poor metallicity range, in agreement with observations. Our results give further support to the idea that stars at very low metallicities could have rotational velocities of the order of $600-800 \mathrm{~km} \mathrm{~s}^{-1}$.

Key words. stars: rotation - Galaxy: evolution

\section{The still unexplored very low metallicity range}

The extremely metal-poor halo stars $([\mathrm{Fe} / \mathrm{H}]<-3, \log (\mathrm{O} / \mathrm{H})+$ $12<7$, Cayrel et al. 2004; Beers \& Christlieb 2005; Spite et al. 2005 , hereafter S05) are not only relics of the earliest phases of the formation of the Milky Way (MW), but also represent the lowest metallicities measured in the universe. So far, high- $z$ observations were not able to find objects in this very low metallicity range. Therefore, it is in the halo of the MW that we have the unique possibility of studying the evolution of abundance ratios at metallicities never explored before.

In particular, until very recently the nitrogen enrichment in low metallicity environments could be studied only through HII region abundances in the outer parts of spiral galaxies or in blue compact galaxies (both showing $\log (\mathrm{O} / \mathrm{H})+12 \gtrsim$ 7, e.g. Izotov et al. 2006) or Damped Lyman Alpha systems (DLAs, $[\mathrm{Fe} / \mathrm{H}]>-2.5$, Wolfe et al. 2005). The very metalpoor halo stars of S05 are unique since they provide the opportunity to study the nitrogen enrichment of the insterstellar medium (ISM) at metallicities never explored before in chemical evolution models ${ }^{1}$. New effects are observed at such low metallicities. Recent measurements of nitrogen abundances in

\footnotetext{
${ }^{1}$ In this Letter we concentrate on the so-called normal very metalpoor stars, since our goal is to explain the mean ISM enrichment at this low metallicity range. Thus, we will not address the carbon-rich-ultrametal-poor stars - see Beers \& Christlieb (2005) for a recent review on this subject.
}

metal-poor stars (S05) show a high N/O ratio suggesting high levels of production of primary nitrogen in massive stars. Moreover, the N/O abundance ratios in metal-poor stars exhibit a large scatter (roughly 1 dex, much larger than their quoted error bars), although none of the stars measured so far has N/O ratios as low as the ones observed in DLAs.

In a recent paper Chiappini et al. (2005, hereafter CMB05) studied the implications of this new data set on our understanding of nitrogen enrichment in the MW. By the time the latter paper was published there was no set of stellar yields able to explain the very metal-poor data of S05. Using the so-called population III stellar yields available in the literature did not solve the problem either (see also Ballero et al. 2006 for chemical evolution models computed with Pop. III stellar yields provided by different authors). In CMB05 it was concluded that the only way to account for the new data was to assume that stars at low metallicity rotate sufficiently fast to enable massive stars to contribute much larger amounts of nitrogen. It was predicted that massive stars born with metallicities below $Z=10^{-5}$ should produce a factor of 10 up to a few times $10^{2}$ more nitrogen (depending on the stellar mass) than the values given by Meynet \& Maeder (2002a, hereafter MM02) for $Z=10^{-5}$ and $v_{\text {rot }}^{\text {ini }}=300 \mathrm{~km} \mathrm{~s}^{-1}$. The physical motivation for this would be an increase in the rotational velocity in very metal-poor stars (Maeder et al. 1999; Meynet et al. 2006) and hence an increase in the nitrogen yields (MM02 - see below). In this framework it is possible to understand the apparently contradictory finding 


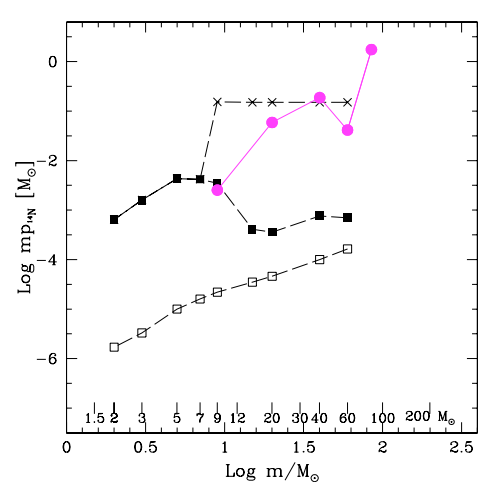

Fig. 1. Stellar yields for ${ }^{14} \mathrm{~N}$ at low metallicities for the whole stellar mass range. The yields of MM02 for stellar models with and without rotation for $Z=10^{-5}$ are shown by filled and open squares, respectively. The asterisks connected by the long-dashed line show the ad hoc stellar yields adopted for $Z<10^{-5}$ in the heuristic model of CMB05 (see text). The dots show the new stellar yields computed for massive stars born at $Z=10^{-8}$. The new computations of Hirschi (2006) for $Z=10^{-8}$ lead to a large increase in $\mathrm{N}$ yields for masses above $\sim 20 M_{\odot}$, similar to the predictions of CMB05. This agreement is striking considering that they were obtained from completely different approaches.

by S05 of a large scatter in N/O and the almost complete lack of scatter in $[\alpha / \mathrm{Fe}]$ ratios of the same very metal-poor halo stars (Cayrel et al. 2004). In fact, the scatter in the N/O abundance ratios could be related to the distribution of the stellar rotational velocities as a function of metallicity.

Whether the above suggestions were physically plausible remained to be assessed by stellar evolution models computed at lower metallicities, which took rotation and mass loss into account. New stellar evolution models have been computed for metallicities $Z=10^{-8}$ (Meynet et al. 2006; Hirschi 2006) for massive stars. The new calculations show that, if the stars at all $Z$ start their lives on the zero age main sequence (ZAMS) with on average a fraction of $\sim 0.5$ the critical velocity, the low $Z$ stars easily reach break-up velocity during MS evolution. Fast rotation also contributes producing a more efficient mixing at lower $Z$, thereby leading to a large production of $\mathrm{N}$ in massive stars. In this Letter we show the impact of these new stellar yields on the chemical enrichment of $\mathrm{C}, \mathrm{N}$, and $\mathrm{O}$ of the earliest phases of the MW. The new stellar yields are presented in Sect. 2. Our results are shown in Sect. 3 and our conclusions drawn in Sect. 4

\section{New yields for very metal-poor massive stars}

This Letter focuses in the very metal-poor range (below $[\mathrm{Fe} / \mathrm{H}] \simeq-3$ or $\log (\mathrm{O} / \mathrm{H})+12 \simeq 7$ ) where no intermediate mass star would have had time to contribute to the ISM enrichment (according to our non-stochastic approach). The effect of rotation on the evolution of low and intermediate mass stars (LIMS) and their impact on the galactic ISM at higher metallicities will be discussed elsewhere. Here we adopt the stellar yields for LIMS given in MM02 but aware of the limitations already discussed in Chiappini et al. (2003).

For massive stars we adopt the new yields shown in Fig. 1. Filled squares show stellar yields of MM02 for their lowest

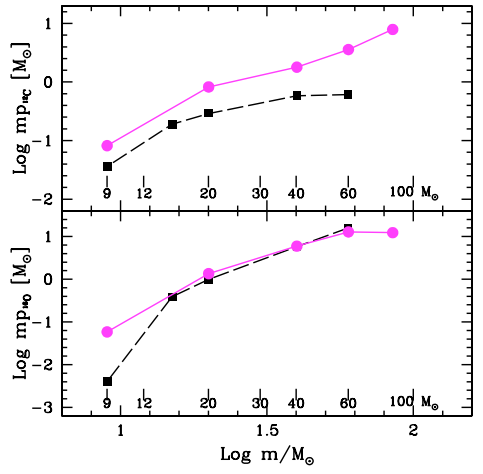

Fig. 2. Stellar yields for ${ }^{12} \mathrm{C}$ (upper panel) and for ${ }^{16} \mathrm{O}$ (lower panel) for massive stars. Curves labeled as in Fig. 1.

metallicity case $\left(Z=10^{-5}\right)$ resulting from models with rotation $\left(v_{\mathrm{rot}}^{\mathrm{ini}}=300 \mathrm{~km} \mathrm{~s}^{-1}\right)$, while open symbols stand for models computed with $v_{\text {rot }}^{\text {ini }}=0 \mathrm{~km} \mathrm{~s}^{-1}$. The asterisks connected by the long-dashed line show the ad hoc stellar yields for metallicities $Z<10^{-5}$ adopted in the heuristic model of CMB05. The dots show the new results obtained by Hirschi (2006; see also Meynet et al. 2006) for $Z=10^{-8}$. The stellar yields for the $Z=10^{-8}$ case were computed according to the following assumption: stars begin their evolution on the ZAMS with approximately the same angular momentum content, regardless of their metallicity. At solar metallicity, observations indicate a mean rotational velocity of a $60 M_{\odot}$ star on the MS of the order of $200 \mathrm{~km} \mathrm{~s}^{-1}$, which corresponds to an initial angular momentum of the order of $2 \times 10^{53} \mathrm{~g} \mathrm{~cm}^{2} \mathrm{~s}^{-1}$ (see Meynet et al. 2006 for details). At a metallicity of $Z=10^{-8}$, this corresponds to $v_{\text {rot }}^{\text {ini }}=800 \mathrm{~km} \mathrm{~s}^{-1}$. In other words, we adopt a rotational velocity such that the ratio between $v_{\text {rot }}^{\text {ini }} / v_{\text {breakup }}$ remains almost constant (around 0.5) with mass and metallicity (see Hirschi 2006 for details). The very interesting result is that the new computations by Hirschi (2006) for $Z=10^{-8}$ predict a large increase in the $\mathrm{N}$ yields for stars above $20 M_{\odot}$, similar to the ad hoc yields of CMB05 (see Fig. 1). Figure 2 shows a comparison of the stellar yields from stellar evolution models with rotation for $Z=10^{-8}\left(v_{\text {rot }}^{\text {ini }}=800 \mathrm{~km} \mathrm{~s}^{-1}\right)$ and $10^{-5}\left(v_{\text {rot }}^{\text {ini }}=300 \mathrm{~km} \mathrm{~s}^{-1}\right)$ for ${ }^{12} \mathrm{C}$ (upper panel) and ${ }^{16} \mathrm{O}$ (lower panel). The carbon yields for the $Z=10^{-8}$ case are systematically higher than the ones at $Z=10^{-5}$ (although by significantly lower factors than in the case of ${ }^{14} \mathrm{~N}$ - see Sect. 3). The differences between the $Z=10^{-8}$ and $10^{-5}$ yields shown in Figs. 1 and 2 are to be ascribed mainly to the different $v_{\text {rot }}^{\text {ini }}$ adopted in each case, the physics involved being essentially the same.

\section{Chemical evolution at very low metallicities}

The adopted chemical evolution model for the MW is the socalled "two-infall model", which is the same model as was used in CMB05. Figure 3 shows our model predictions for the evolution of nitrogen and carbon for different assumptions of stellar yields at low metallicity. The solid curves show a model computed with MM02 stellar yields (as in Chiappini et al. $2003^{2}$ ). This model was computed under the assumption that the $Z=10^{-5}$ yield table $\left(v_{\text {rot }}^{\text {ini }}=300 \mathrm{~km} \mathrm{~s}^{-1}\right)$ would be valid

\footnotetext{
2 For the solar metallicity we are adopting the new calculations presented in Hirschi et al. (2004).
} 


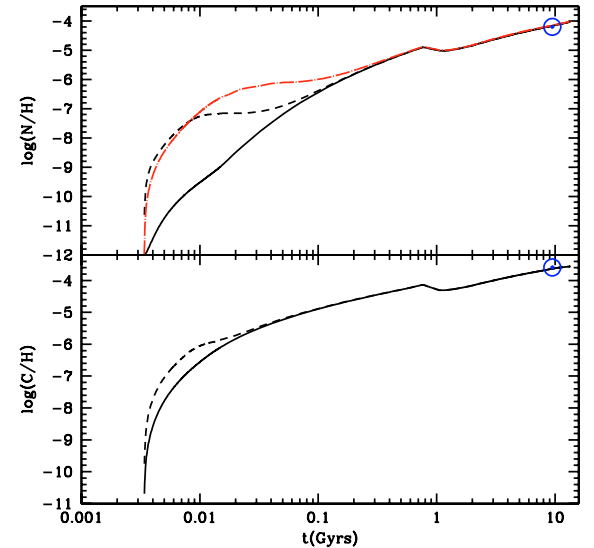

Fig. 3. Predicted evolution of nitrogen (upper panel) and carbon (lower panel) according to a chemical evolution model computed with different stellar yield sets for metallicities below $Z=10^{-5}$ : a) solid line - a model computed under the assumption that the lowest metallicity yield table of MM02 $\left(Z=10^{-5}\right)$ is valid down to $Z=0$; $\left.\mathbf{b}\right)$ dotdashed line (red in the online version) - the model of CMB05 where an ad hoc higher yield of nitrogen is assumed for metallicities below $10^{-5}$; c) dashed line - the new model presented in this Letter adopting new stellar yields computed by new stellar evolution models with faster rotation for $Z=10^{-8}$ massive stars.

down to $Z=0$. As can be seen in Fig. 4, this model cannot explain the high levels of $\mathrm{N} / \mathrm{O}$ or the $\mathrm{C} / \mathrm{O}$ upturn observed in the very metal-poor halo stars of $\mathrm{S}^{3} 5^{3}$. The dot-dashed line shows the heuristic model of CMB05. This model is the same as the one represented by the solid line except that for metallicities $\mathrm{Z}<10^{-5}$ the yields of nitrogen were strongly increased in comparison to the ones given in MM02 for massive stars (the adopted yields in the case of this model are shown in Fig. 1 by the asterisks connected by a long-dashed line). As a consequence, this model produces more nitrogen at the beginning of galaxy evolution (as shown in Fig. 3, upper panel, dot-dashed curve), leading to large N/O ratios at low metallicities (Fig. 4, upper panel, dot-dashed curve). However, it was unclear whether stellar evolution models at such low metallicities could predict such a large enhancement of nitrogen and what would be the impact for $\mathrm{C}$ and $\mathrm{O}$.

The dashed curves in Figs. 3 and 4 show our most recent model computed with the new stellar yields at $Z=10^{-8}$ of Hirschi (2006) for massive stars, assuming them to be valid down to $Z=0^{4}$. The evolution of nitrogen predicted by this model (dashed line in Fig. 3, upper panel) is similar to the one predicted by the CMB05 model (dot-dashed line), except for the later times (as expected since the ad hoc yields of CMB05 are higher than the ones of Hirschi for masses below $20 M_{\odot}$ ).

\footnotetext{
${ }^{3}$ One of the main assumptions when comparing chemical evolution predictions with abundance data is that they represent the pristine abundances from the ISM, from which the stars formed. Therefore, objects that could have undergone mixing processes should be avoided. The data shown here are in principle unmixed stars (S05).

4 The physics adopted in the $Z=10^{-8}$ models should be valid down to $Z \sim 10^{-10}$, which represents the metallicity limit below which massive stars first enter the phase of H-burning via the pp chain, followed by the $3 \alpha$ reaction, which then allows the $\mathrm{CNO}$ cycle to proceed, as in $Z=0$ (Pop. III) stars.
}

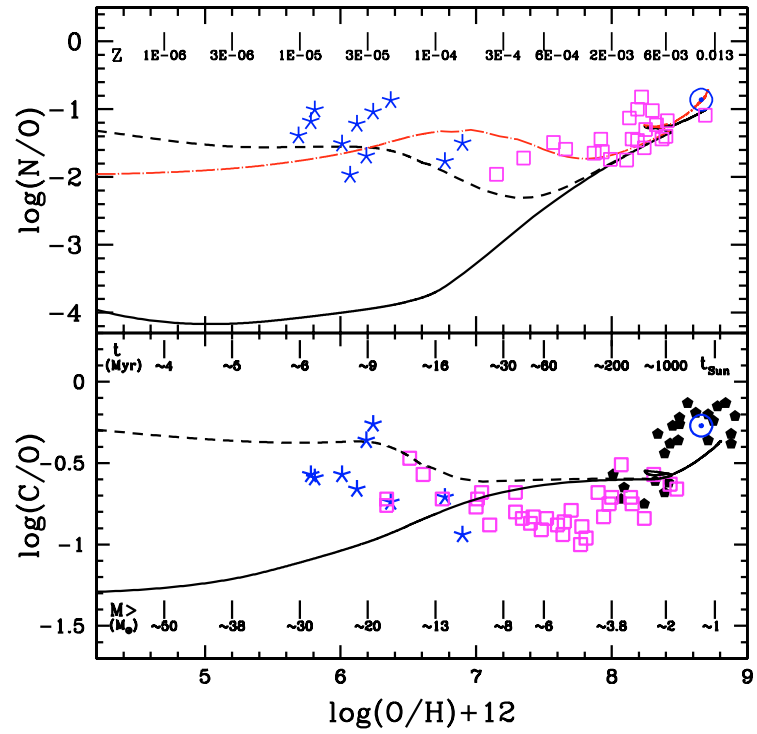

Fig. 4. Upper panel: solar vicinity diagram $\log (\mathrm{N} / \mathrm{O})$ vs. $\log (\mathrm{O} / \mathrm{H})+12$. The data points are from Israelian et al. (2004 large squares), S05 (asterisks). Models are labeled as in Fig. 3. Lower panel: solar vicinity diagram $\log (\mathrm{C} / \mathrm{O})$ vs. $\log (\mathrm{O} / \mathrm{H})+12$. The data are from Spite et al. (S05 - asterisks), Israelian et al. (2004 - squares), Nissen (2004 - filled pentagons). Solar abundances (Asplund 2005, and references therein) are also shown.

The similarity of both curves is striking as they were obtained following completely independent approaches. This result implies that faster rotation is able to account for the S05 observations. Some differences are also seen for the $\mathrm{C}$ evolution (see Fig. 3, lower panel). Our new model predicts a $\mathrm{C} / \mathrm{O}$ upturn at low metallicities (Fig. 4, lower panel, dashed curve). This upturn results from the strong production of primary nitrogen. Indeed, high production of primary nitrogen implies a very active H-burning shell, which contributes a large part of the total luminosity of the star. As a consequence, part of the total luminosity compensated by the energy produced in the helium core is reduced, making the average core temperature and thus the efficiency of the ${ }^{12} \mathrm{C}(\alpha, \gamma){ }^{16} \mathrm{O}$ reaction lower. More efficient mixing also leads to greater mass loss, decreasing the He-core size. Higher $\mathrm{C} / \mathrm{O}$ ratios are thus obtained at the end of the $\mathrm{He}-$ burning phase.

We note that the effects obtained in this Letter, namely, the high $\mathrm{N} / \mathrm{O}$ and the $\mathrm{C} / \mathrm{O}$ upturn at very low metallicities can be explained without invoking Pop. III stars (i.e. without changing the IMF or including zero-metallicity stellar yields) and hence do not necessarily imply the signature of Pop. III stars as previously claimed in the literature (e.g. Akerman et al. 2004). The only shortcoming of the present model is that it predicts slightly higher $[\mathrm{C} / \mathrm{Fe}]$ with respect to the observations of S05 (around 0.3 dex larger at $[\mathrm{Fe} / \mathrm{H}] \sim-3$ up to 0.9 dex at $[\mathrm{Fe} / \mathrm{H}] \sim-4)$. However, in this case there are two large uncertainties, namely a) the stellar yields for Fe in massive stars (strongly dependent on the adopted mass cut) and b) the $[\mathrm{C} / \mathrm{Fe}]$ ratios of $\mathrm{S} 05$ could still be affected by uncertainties due to the first dredgeup dilution of $C$ on the giant branch (Iben 1965) and NLTE/3D corrections. 
Intermediate mass stars of this same low metallicity could also produce large amounts of nitrogen, if this production is linked to high rotational velocities. In this case, a flatter curve in the N/O diagram would be obtained. In fact, as can be seen in Fig. 4, the agreement between model predictions for $\mathrm{N} / \mathrm{O}$ and $\mathrm{C} / \mathrm{O}$ observations worsens at $\log (\mathrm{O} / \mathrm{H})+12>7$, where the AGB contribution starts to be effective. Super AGB stars, not included in the present Letter, could also contribute to the $\mathrm{N}$ enrichment of the ISM (Siess, priv. comm.). Stellar evolution models with rotation for LIMS computed up to the final evolutionary phases are being developed and will soon be available to be incorporated in chemical evolution models.

\section{Discussion and conclusions}

In this Letter, we computed chemical evolution models adopting the very recent calculations of Hirschi (2006) for the evolution of massive stars at very low metallicities under the assumption of an almost constant ratio $v_{\text {rot }}^{\text {ini }} / v_{\text {breakup }}$ as a function of metallicity (i.e. where the $v_{\text {rot }}^{\text {ini }}$ increases towards lower metallicities). In such a framework, massive stars can produce large amounts of nitrogen (of the order of the ones suggested in CMB05).

At present, this is the only way to explain the high nitrogen abundances measured recently in normal halo stars (S05). This gives further support to the idea that stars rotate faster at very low metallicities. The new stellar evolution models also produce some extra carbon at $Z \leq 10^{-5}$. As a consequence, an upturn is produced in the $\mathrm{C} / \mathrm{O}$ at $\log (\mathrm{O} / \mathrm{H})+12<7$ metallicities. This result is obtained without the need of introducing Pop. III stars. Finally, our results show that the $\mathrm{C}$ and $\mathrm{N}$ yields strongly depend on metallicity for $Z \leq 10^{-5}$, once rotation is taken into account. This has a strong impact on the chemical enrichment of the very metal-poor ISM. It is thus important to avoid extrapolating the stellar yields in this metallicity range when computing chemical evolution models, even if the timespan to reach $Z=10^{-5}$ is just a few Myrs.

The only alternative explanation of the observations considered in this Letter would be if AGB stars contributed to the ISM enrichment before $[\mathrm{Fe} / \mathrm{H}] \sim-3$. This would mean that the timescales for the chemical evolution of the halo would be very different from the ones adopted here. Our model assumes that the halo was formed by an exponential infall with an e-folding time of $\sim 1$ Gyr. The enrichment timescales do not change if the infall timescale is increased by a factor of 2 (nor if the star formation efficiency is divided by 2). Models assuming outflows could delay the ISM enrichment so that AGBs would have time to contribute. However, in this case it should be seen if such models could still fit the halo abundance ratios well. The chemical evolution model presented here (without outflows) also agrees well with several other abundance ratios (François et al. 2004).

Here we show the results for the mean ISM enrichment history, assuming that at a given metallicity all stars of a given mass rotate at the same velocity. Further computations are in progress, and we envision being able to compute chemical evolution models in which a distribution of rotational velocities is assumed for each metallicity. These models would predict a scatter in $\mathrm{N} / \mathrm{O}$ to be compared to the observed one (forthcoming paper).

\section{Acknowledgements. INAF/PRIN CRA 1.06.08.02.}

\section{References}

Akerman, L. J., Carigi, L., Nissen, P. E., et al. 2004, A\&A, 414, 931 Asplund, M. 2005, ARA\&A, 43, 481

Ballero, S., Matteucci, F., \& Chiappini, C. 2006, New Astron., 11, 306 Beers, T. C., \& Christlieb, N. 2005, ARA\&A, 43, 531

Cayrel, R., Depagne, E., Spite, M., et al. 2004, A\&A, 416, 1117

Chiappini, C., Matteucci, F., \& Meynet, G. 2003, A\&A, 410, 257

Chiappini, C., Matteucci, F., \& Ballero, S. K. 2005, A\&A, 437, 429 (CMB05)

Ekström, et al. 2006, in preparation

François, P., Matteucci, F., Cayrel, R., et al. 2004, A\&A, 421, 613

Hirschi, R., Meynet, G., \& Maeder, A. 2004, A\&A, 425, 649

Hirschi, R. 2006, in preparation

Iben, I. Jr. 1965, ApJ, 142, 1447

Israelian, G., Ecuvillon, A., Rebolo, R., et al. 2004, A\&A, 421, 649

Izotov, Y. I., Stasinska, G., Meynet, G., et al. 2006, A\&A, 448, 955

Maeder, A., Grebel, E. K., \& Mermilliod, J.-C. 1999, A\&A, 346, 459

Meynet, G., \& Maeder, A. 2002a, A\&A, 390, 561 (MM02)

Meynet, G., \& Maeder, A. 2002b, A\&A, 381, L25

Meynet, G., Ekström, S., \& Maeder, A. 2006, A\&A, 447, 623

Nissen, P. E. 2003, in CNO in the Universe, ed. C. Charbonnel, D. Schaerer, \& G. Meynet, ASP Conf. Ser., 304, 60

Spite, M., Cayrel, R., Plez, B., et al. 2005, A\&A, 430, 655 (S05)

Wolfe, A. M., Gawiser, E., \& Prochaska, J. X. 2005, ARA\&A, 43, 861 